\title{
HOMOTOPY OPERATIONS UNDER A FIXED SPACE BY
}

\author{
D. E. KRUSE AND J. F. MCCLENDON
}

\begin{abstract}
The problem of classifying extensions of a function up to relative homotopy leads in a natural way to the homotopy operations of the title. The operations, stable and unstable, primary and higher order, are defined and studied. Some specific applications are worked out.
\end{abstract}

Let $f: A \rightarrow Y$ be a map of topological spaces and $X \supset A$. If $g, g^{\prime}: X \rightarrow Y$ both extend $f$ then they are homotopic relative to $A$ if there is a homotopy $H$ : $X \times I \rightarrow Y$ with $H_{0}=g, H_{1}=g^{\prime}$ and $H(a, t)=f a$ all $a \in A$. Let $[X, Y]^{A}$ (or $[X, Y]^{A} f$ ) denote the set of equivalence classes, which is nonempty if and only if $f$ actually extends. We are ultimately interested in studying this set, especially in a situation where $X$ is known and certain properties of $Y$ are given (e.g. vanishing of some Whitehead products). Certain homotopy operations arise in a natural way in this problem and the main purpose of this paper is to define these operations and develop their properties. In the last section we do state, without proof, two special cases of a general theorem about $[X, Y]^{A}$. We use the operations to compute answers in special cases. Operations can always be thought of as spectral sequence differentials, so the paper could be viewed as a study of differentials from spectral sequences naturally associated with $[X, Y]^{A}$-however we do not take that view here.

In $\$ 0$ we make a few observations of a categorical nature. These provide a little perspective for twisted operations and may be of interest to someone wishing to work in a different category, or even categorically. In $\$ 1$ we give notation and geometric definitions. $\$ 2$ treats a suspension operation. $\S \S 3,4$ and 5 treat primary and secondary twisted operations. $\$ 6$ gives the applications mentioned above.

0. General comments. Suppose $\mathcal{C}, \mathcal{C}^{\prime}$ and $\mathcal{Q}$ are categories and $\beta: \mathcal{C}^{\prime} \rightarrow \mathcal{C}_{\text {a }}$ fixed functor. Think of $\mathcal{C}$ as a known smaller category and $\mathcal{C}^{\prime}$ a larger, less known, category. We want to investigate $H: \mathcal{C}^{\prime} \rightarrow \mathbb{Q}$. Note that if $F: \mathcal{C} \rightarrow \mathbb{Q}$ we get $F^{\prime}: \mathcal{C}^{\prime} \rightarrow \mathbb{Q}, F^{\prime}(Z)=F(\beta Z)$, and if $\theta: F^{\prime} \rightarrow G^{\prime}$ is a natural transformation of such functors we have $\theta: F(\beta Z) \rightarrow G(\beta Z)$. So $\theta$ can be viewed as a twisted natural transformation from $F$ to $G$. Ideally we would like a filtration for $H(Z)$ with quotients of the form $F_{n}^{\prime}(Z)=F_{n}(\beta Z)$ (since these

Received by the editors December 2, 1975.

AMS (MOS) subject classifications (1970). Primary 55G37, 55G35; Secondary 55D05. 
are more known)-next best is to get quotients in terms of kernels and cokernels of twisted operations defined on these functors. This is what we do in the present paper (see §6). To be slightly more specific use $H(Z)=$ $\operatorname{Hom}^{\prime}(T, Z), T \in \mathcal{C}^{\prime}$. Suppose $\beta$ has an adjoint $\alpha$ and the filtration gives quotients in terms of $\operatorname{Hom}^{\prime}\left(\alpha T_{n}, Z\right), T_{n} \in \mathcal{C}$. Then the quotients are in terms of $\operatorname{Hom}\left(T_{n}, \beta Z\right)$ and these are the $F_{n}$ 's.

Let $\mathcal{C}$ be any category and $C \in \mathcal{C}$. Then we can form $\mathcal{C}^{\prime}=\mathcal{C}(C \rightarrow 0)=$ all pairs $(X, \check{x}), \check{x}: C \rightarrow X$. If $\mathcal{C}$ has sums then we can form $X+C$ and $(C \rightarrow X+C)$ is an object in $C^{\prime}$. Let $\alpha: C \rightarrow C^{\prime}, \alpha X=(C \rightarrow X+C), \beta$ : $C^{\prime} \rightarrow C, \beta$ the forgetful functor. Then the description above applies.

For simplicity's sake we work only with $C=T o p(*)$ (or pairs) and $T_{n}=$ $S^{n}$. However, much of what is done can easily be done in terms of natural transformations of functors (as above), especially representable covariant functors-more can be done in terms of $\mathcal{C}(C \rightarrow 0)$ - and virtually everything carries over to functors $[\Sigma(X, A),(Y, B)]-$ e.g. to homotopy with coefficients.

Even in the case $C=$ point, the untwisted case, our treatment of homotopy operations is somewhat different from the usual treatments.

1. Terminology and some properties of $\bar{\Sigma}$. In this section we give the basic geometric definitions that we will use, both for the topological category, and the related category $\operatorname{Top}(C \rightarrow D)$. By making even the elementary definitions explicit we hope to avoid ambiguities, particularly in regard to sign. Proofs are not difficult and most will be omitted.

For simplicity we will assume at the outset that all spaces have base points. $K_{u} X=X \times I / X \times 0$ and $\Sigma_{u} X=K_{u} X / X \times 1$ are the unreduced cone and suspension of the space $X . K X=K_{u} X / * \times I$ and $\Sigma X=\Sigma_{u} X / * \times I$ are the reduced cone and suspension of $X . r=r(X):\left(K_{u} X, X\right) \rightarrow(K X, X)$ is the natural projection with $\bar{r}=\bar{r}(X):(K X, X) \rightarrow\left(K_{u} X, X\right)$ a fixed pointed homotopy inverse. (Whenever $r$ is used, assume the base point of $X$ is nondegenerate.) $s=s(X): \Sigma_{u} X \rightarrow \Sigma X$ and $\bar{s}$ are similarly defined ( $\bar{s}$ defined using $r) . c_{u}(X):\left(K_{u} X, X\right) \rightarrow\left(\Sigma_{u} X, *\right), c(X):(K X, X) \rightarrow(\Sigma X, *)$ are the natural quotient maps $K_{u}^{-} X, K_{u}^{+} X \subset \Sigma_{u} X$ are the images of $X \times\left[0, \frac{1}{2}\right]$, $X \times\left[\frac{1}{2}, 1\right]$ respectively and $i^{-}: K_{u} X \rightarrow K_{u}^{-} X$ is defined from $(x, t) \rightarrow$ $(x, t / 2)$ and $i^{+}: K_{u} X \rightarrow K_{u}^{+} X$ from $(x, t) \rightarrow(x, 1-t / 2)$. The reduced constructions are done similarly. $f: X \rightarrow Y$ gives

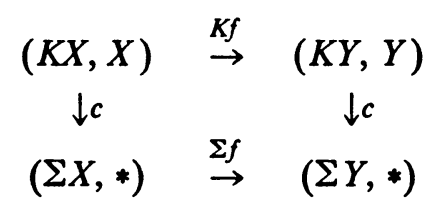

and similarly for $K_{u}, C_{u}$ and $\Sigma_{u}$. Also $r c_{u}=c r, c_{u} \bar{r} \sim \bar{r} c$.

Define $E^{n}=\left\{x \in \mathbf{R}^{n}|| x \mid<1\right\}, S^{n-1}=\left\{x \in \mathbf{R}^{n}|| x \mid=1\right\}, a_{u}=a_{u}(n)$ : 
$\left(K_{u} S^{n-1}, S^{n-1}\right) \rightarrow\left(E^{n}, S^{n-1}\right), a_{u}(x, t)=t x, a_{u}$ is a homeomorphism. $a=$ $a(n)=a_{u}(n) \bar{r}:\left(K S^{n-1}, S^{n-1}\right) \rightarrow\left(E^{n}, S^{n-1}\right)$, a homotopy equivalence.

Let $E_{+}^{n}=\left\{x \in S^{n} \mid X_{n+1}>0\right\}, E_{-}^{n}=\left\{x \in S^{n} \mid x_{n+1}<0\right\}, \pi_{+}: E_{+}^{n} \rightarrow E^{n}$, $\pi_{-}: E_{-}^{n} \rightarrow E^{n}$ the projections. Define $e_{u}=e_{u}(n+1): \Sigma_{u} S^{n} \rightarrow S^{n+1}$ by $e_{u}=$ $\pi_{+}^{-1} a_{u} i_{+}^{-1} \cup \pi_{-}^{-1} a_{u} i_{-}^{-1}: \quad \Sigma_{u} S^{n}=K_{u}^{+} S^{n} \cup K_{u}^{-} S^{n} \rightarrow E_{+}^{n+1} \cup E_{-}^{n+1}=S^{n+1}$, and $e=e(n+1): \Sigma S^{n} \rightarrow S^{n+1}, e=e_{u} \bar{r}$, so $e$ is a pointed homotopy equivalence ( $\Sigma S^{n}$ and $S^{n+1}$ are actually homeomorphic-but we ignore this). Now define $c_{u}:\left(E^{n}, S^{n-1}\right) \rightarrow\left(S^{n}, *\right)$ by $c_{u}=e_{u} c_{u}\left(S^{n-1}\right) a^{-1}$. Note that the following diagram is homotopy commutative

$$
\begin{array}{ccc}
\left(K S^{p}, S^{p}\right) & \stackrel{a}{\rightarrow} & \left(E^{p+1}, S^{p}\right) \\
\downarrow c\left(S^{p}\right) & & \downarrow c_{u}(p+1) \\
\left(\Sigma S^{p}, *\right) & \stackrel{e}{\rightarrow} & \left(S^{p+1}, *\right)
\end{array}
$$

Now for $f: S^{p} \rightarrow S^{n}$ define $s f: S^{p+1} \rightarrow S^{n+1}, k f:\left(E^{p+1}, S^{p}\right) \rightarrow\left(E^{n+1}, S^{n}\right)$ by

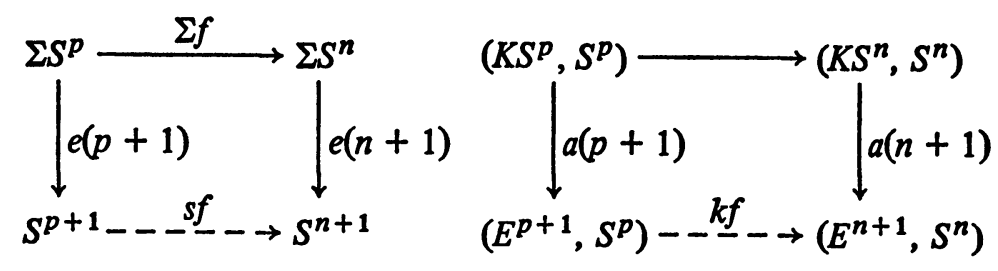

Thus the following diagram is homotopy commutative

$$
\begin{array}{ccc}
\left(E^{p+1}, S^{p}\right) & \stackrel{k f}{\rightarrow} & \left(E^{n+1}, S^{n}\right) \\
\downarrow c_{p+1} & & \downarrow c_{n+1} \\
\left(S^{p+1}, *\right) & \stackrel{s f}{\rightarrow} & \left(S^{n+1}, *\right)
\end{array}
$$

If $f_{i}: X \rightarrow Y_{i}$ then $f=\left(f_{1}, \ldots, f_{n}\right): X \rightarrow Y_{1} \times \cdots \times Y_{n}$ sends $x$ to $\left(f_{1}(x), \ldots, f_{n}(x)\right)$. If $g: X_{i} \rightarrow Y$ then $g=\left\langle g_{1}, \ldots, g_{n}\right\rangle X_{1} \vee \cdots \vee X_{n} \rightarrow Y$, $g$ restricted to $X_{i}$ is $g_{i}$.

We will follow [J. H. C. Whitehead, 15, §4] and use an axiomatic boundary operator until we explicitly state otherwise. All spaces, maps, pairs, etc. are base pointed. The base point of $S^{n-1}$ and $E^{n}$ is $(1,0, \ldots, 0)$. [ $\left.(P, Q),(X, A)\right]$ is the set of base pointed homotopy classes of maps from $(P, Q)$ to $(X, A)$.

$$
\begin{aligned}
\pi_{n}(X, A) & =\left[\left(E^{n}, S^{n-1}\right),(X, A)\right], \\
\pi_{n}(X) & =\pi_{n}(X, *)=\left[\left(E^{n}, S^{n-1}\right),(X, *)\right] .
\end{aligned}
$$

A homotopy boundary operator is a sequence $\partial=\left(\partial_{1}, \partial_{2}, \ldots\right)$ of natural transformations of functors such that the homotopy sequence of each pair is exact. 


$$
\begin{aligned}
\lambda_{n+1} & =[\mathrm{id}] \in \pi_{n+1}\left(E^{n+1}, S^{n}\right), \\
\mu_{n} & =\partial \lambda_{n+1} \in \pi_{n}\left(S^{n}\right) \text { note } \partial[F]=\left(F \mid S^{n}\right)_{*} \mu_{n} .
\end{aligned}
$$

The suspension $\Sigma: \pi_{n}(X) \rightarrow \pi_{n+1}(\Sigma X)$ is defined by

$$
\Sigma=c_{*} \partial^{-1}: \pi_{n}(X) \leftarrow \pi_{n+1}(K X, X) \rightarrow \pi_{n+1}(\Sigma X) .
$$

1.1 LeMma. $(\Sigma f)_{*}(\Sigma w)=\Sigma\left(f_{*} w\right)$.

We have $\Sigma: \pi_{k-1}\left(S^{k-1}\right) \rightarrow \pi_{k}\left(S^{k}\right)$. Choose $h_{k}: \Sigma S^{k-1} \rightarrow S^{k}$ such that $h_{*} \Sigma\left(\mu_{k-1}\right)=\mu_{k}$. Consider now $c_{n^{*}}, \mu_{n^{*}}: \pi_{n}\left(E^{n}, S^{n-1}\right) \rightarrow \pi_{n}\left(S^{n}\right)$. Both are isomorphic so they differ by +1 or -1 . We define $u:\{1,2, \ldots\} \rightarrow\{+1$, $-1\}, u=u(\partial)$, by $\left(\mu_{n}\right)_{*}=u(n)\left(c_{n}\right)_{*}$ or $\mu_{n} \sim \bar{u}(n) c_{n}, c_{n} \sim \bar{u}(n) \mu_{n}$. Here $\bar{u}(n):$ $S^{n} \rightarrow S^{n}$ is a map of degree $u(n)$.

1.2 LEMMA. $h_{k}=\bar{u}(k) e(k)$.

Now define $E=\left(\mu_{p+1}\right)_{*} \partial^{-1}: \pi_{n}\left(S^{p}\right) \rightarrow \pi_{n+1}\left(S^{p+1}\right)$.

$$
\pi_{n}\left(S^{p}\right) \leftarrow \pi_{n+1}\left(E^{p+1}, S^{p}\right) \rightarrow \pi_{n+1}\left(S^{p+1}\right) .
$$

1.3 Lemma. (a) $f: S^{m} \rightarrow S^{p}, E\left(f_{*} \alpha\right)=u(m+1) u(p+1) s(f)_{*} E(\alpha)$

(b) $E=\left(h_{p+1}\right)_{*} \Sigma$ (so $E$ will often be written as $\Sigma$ ).

In the following lemma, $k:\left[S^{p-1}, S^{n-1}\right] \rightarrow\left[\left(E^{p}, S^{p-1}\right),\left(E^{n}, S^{n-1}\right)\right]$ is used as in the diagrams several paragraphs above.

1.4 LEMMA. $\partial: \pi_{p}\left(E^{n}, S^{n-1}\right) \rightarrow \pi_{p-1}\left(S^{n-1}\right), \partial\left(k\left(\mu_{p-1}^{*-1} \alpha\right)\right)=\alpha$.

Now define $\pi_{n}^{\prime}(X)=\left[S^{n}, X\right]$ and note the isomorphism

$$
\mu_{n}^{*}: \pi_{n}^{\prime}(X)=\left[S^{n}, X\right] \rightarrow\left[\left(E^{n}, S^{n-1}\right),(X, *)\right]=\pi_{n}(X) .
$$

1.5 Lemama. (1) $f: X \rightarrow Y, f_{*}: \pi_{n}^{\prime}(X) \rightarrow \pi_{n}^{\prime}(Y)$. Then $f_{*}[g]=[f g]$.

(2) Consider

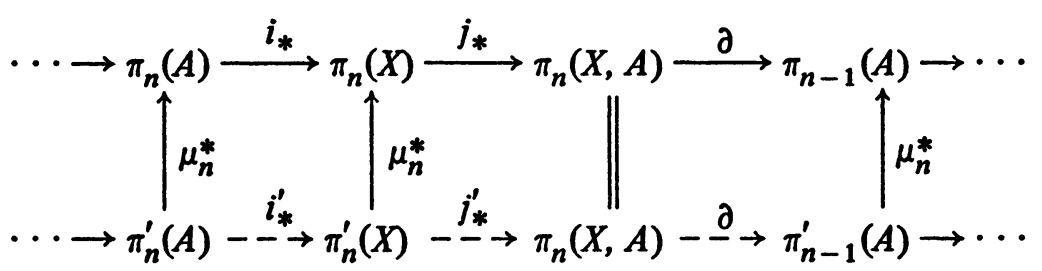

where $i_{*}^{\prime}, j_{*}^{\prime}$ and $\partial^{\prime}$ are defined by the diagram. By (1) $i_{*}^{\prime}[g]=[i g]$. Also $\partial^{\prime}[F]=\left[F \mid S^{n-1}\right], j_{*}^{\prime}[h]=\left[j \circ h \circ \mu_{n}\right]$.

We will frequently identify $\pi_{n}(X)$ and $\pi_{n}^{\prime}(X)$. The identification will always be by means of $\mu^{*}$ and the "'" will be omitted.

We recall from [9, pp. 191-199] that if $u: C \rightarrow D$ is a morphism in a category $T$ then there is a category $T(u: C \rightarrow D)$. The objects are triples 
$(X, \check{x}, \hat{x})$ where $\check{x}: C \rightarrow X, \hat{x}: X \rightarrow D$ and $\hat{x} \check{x}=u$. Morphisms are $T$ morphisms $f: X \rightarrow Y$ such that $f \ddot{x}=\check{y}$ and $\hat{y} f=\hat{x}$. If $u=\mathrm{id}: C \rightarrow C$ write $T(C)$ for $T(u)$. Here $T$ will be $\operatorname{Top}(*)=$ the category of pointed spaces and maps and we will need only

$$
\operatorname{Top}(C)=\operatorname{Top}(\mathrm{id}: C \rightarrow C) \text { and } \operatorname{Top}^{C}=\operatorname{Top}(C \rightarrow *) .
$$

In this section we will work only with $\operatorname{Top}(C)$. All of the usual homotopy constructions of $\operatorname{Top}(*)$ can be carried out in $\operatorname{Top}(C)$ (see [9, §§II.3, II.4]). We will denote the cone and suspension functors of $\operatorname{Top}(C)$ by $\bar{K}$ and $\bar{\Sigma}$. Thus $\bar{K} X=X \times I / R$ where $R$ is the equivalence relations generated by $(x, 0) \sim(\check{x} \hat{x}(x), 0), x \in X$, and $(\check{x}(c), t) \sim\left(\check{x}(c), t^{\prime}\right)$ for $c \in C, \bar{\Sigma} X=\bar{K} X / S$ where $S$ is the equivalence relation generated by $(x, 1) \sim(\check{x} \hat{x} x, 1)$.

1.6 Definition. Let $Y \in \operatorname{Top}(C)$. Define $\bar{\Sigma}: \pi_{n}(Y, C) \rightarrow \pi_{n+1}(\bar{\Sigma} Y, C)$ by

$$
\bar{\Sigma}=k_{*} \partial^{-1} \pi_{n}(Y, C) \leftarrow \pi_{n+1}(\bar{K} Y, Y) \rightarrow \pi_{n+1}(\bar{\Sigma} Y, C) .
$$

Here $k$ is the quotient map, $\partial$ is the boundary homomorphism of the triple $\bar{K} Y, Y, C$. It is an isomorphism since $\pi_{i}(\bar{K} Y, C)=0$ for all $i$.

$\bar{\Sigma}$ is related to the suspension for maps in Top $C$ (see [7], [8]) but the present formulation suffices for the results of this paper. $\bar{\Sigma}$ is clearly natural for maps of $\operatorname{Top}(C)$. In order to state a composition theorem for $\bar{\Sigma}$ we define $\Sigma: \pi_{j}\left(E^{p}, S^{p-1}\right) \rightarrow \pi_{j+1}\left(E^{p+1}, S^{p}\right)$ by

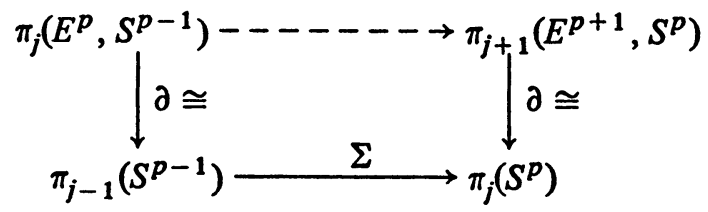

1.7 THEOREM. $\bar{\Sigma}(y \circ u)=(\bar{\Sigma} y) \circ(\Sigma u), y \in \pi_{p}(Y, C), u \in \pi_{n}\left(E^{p}, S^{p-1}\right)$.

We will discuss $\operatorname{Top}(C)$ homotopy operations later. Since $u$ in 1.7 can be viewed as a $\operatorname{Top}(*)$ homotopy operation we can now state:

1.8 Corollary. Let $\Gamma$ be a stable relative homotopy operation for Top*. Then $\bar{\Sigma} \Gamma=\Gamma \bar{\Sigma}\left(\right.$ i.e. $\left.\bar{\Sigma} \Gamma(Y, C)=\Gamma(\bar{\Sigma} Y, C) \bar{\Sigma}: \pi_{n}(Y, C) \rightarrow \pi_{p+1}(\bar{\Sigma} Y, C)\right)$ provided that $Y \in \operatorname{Top}(C)$.

1.9 TheOREM. Suppose $Y \in \operatorname{Top}(C)$ and $\check{y}: C \rightarrow Y$ a cofibration in Top. Suppose $\pi_{q}(Y, C)=0, q<n$. Then $\bar{\Sigma}: \pi_{q}(Y, C) \rightarrow \pi_{q+1}(\bar{\Sigma} Y, C)$ is isomorphic for $q<2 n$, epimorphic for $q \leqslant 2 n+1$.

Proof. This follows from the homotopy excision theorem (Blakers and Massey [3] or Spanier [12]) just as for the case $c=*$.

More generally one can define $\bar{\Sigma}: \pi_{q}\left(Y, Y_{0}\right) \rightarrow \pi_{q+1}\left(\bar{\Sigma} Y, \bar{\Sigma} Y_{0}\right)$ (e.g. by 
using triad groups-or other ways) for $\left(Y, Y_{0}\right) \in \operatorname{Top}(C)$. Assume $Y_{0} \subset Y$ a Top $(C)$ cofibration and $y: C \rightarrow Y_{0}$ a Top cofibration. Assume $\left(Y, Y_{0}\right) n$ connected and $\left(Y_{0}, C\right) m$-connected and get $\bar{\Sigma}$ isomorphic for $q<n+$ $\min (n, m)$ and epimorphic for $q \leqslant n+\min (n, m)+1$.

Note that $\bar{\Sigma}(C \vee Z)=C \vee \Sigma Z$. Also we have

$\left(1 \vee h_{n+1}\right)_{*} \bar{\Sigma}: \pi_{i}\left(C \vee S^{n}, C\right) \rightarrow \pi_{i+1}\left(C \vee \Sigma S^{n}, C\right) \rightarrow \pi_{i+1}\left(C \vee S^{n+1}, C\right)$

and this composition will often be written simply as $\bar{\Sigma}$. If $i=n$ we have $\bar{\Sigma}\left(i_{2}\right) \mu_{n}=i_{2^{*}} \mu_{n+1}$ or $\bar{\Sigma} i_{2^{*} l_{n}}=i_{2^{*} l_{n+1}}$ (depending on whether $\pi_{n}\left(S^{n}\right)$ is viewed as $\left[\left(E^{n}, S^{n-1}\right),\left(S^{n}, *\right)\right]$ or as $\left[S^{n}, S^{n}\right], \iota_{n}=$ [id]). Usually we will write simply $\bar{\Sigma} \mu_{n}=\mu_{n+1}$ or $\bar{\Sigma}_{\iota_{n}}=\iota_{n+1}$.

1.10 Corollary. $\bar{\Sigma}: \pi_{i}\left(C \vee S^{n}, C\right) \rightarrow \pi_{i+1}\left(C \vee S^{n+1}, C\right)$ is isomorphic for $i<2 n-2$ and epimorphic for $i \leqslant 2 n-1$.

2. Further properties of $\bar{\Sigma}$. In this section we prove a theorem relating $\bar{\Sigma}$ and the Whitehead product. We then use $\bar{\Sigma}$ to give a proof of a theorem of Barcus and Barrat.

It will be convenient here to change the domain of $\bar{\Sigma}$. Let $Y \in \operatorname{Top}(C)$. We have

$$
0 \rightarrow \pi_{n}(C) \underset{\dot{y}_{*}}{\stackrel{\hat{y}_{*}}{\leftrightarrows}} \pi_{n}(Y) \underset{\dot{j}_{*}}{\stackrel{\nu}{\leftrightarrows}} \pi_{n}(Y, C) \rightarrow 0
$$

Here $v$ is the injection defined by $v u^{\prime}=u$ where $j_{*} u=u^{\prime}$ and $\hat{y}_{*} u=0$. Then $j_{*} \mid \operatorname{Ker}\left(\hat{y}_{*}\right)$ is an isomorphism of $\operatorname{Ker} \hat{y}_{*}$ and $\pi_{n}(Y, C)$. Here is a diagram displaying both versions of $\bar{\Sigma}$.

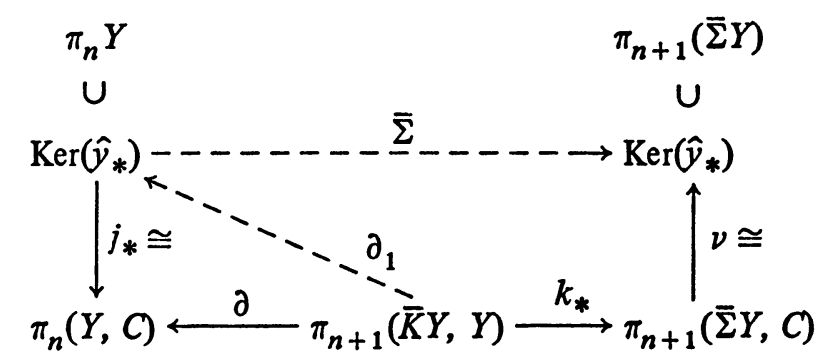

Note that $\partial_{1}: \pi_{n+1}(\bar{K} Y, Y) \rightarrow \pi_{n}(Y)$ has image $\operatorname{Ker}\left(\hat{y}_{*}\right)$ and that $\partial^{-1} \circ\left(j_{*} \mid \operatorname{Ker}\left(\hat{y}_{*}\right)\right)=\partial_{1}^{-1}$. Theorem 1.7 is now valid for $u \in \pi_{n}\left(S^{p}\right)$ also.

For the rest of this section we will use the boundary operator used by J. H. C. Whitehead [15] and Blakers and Massey [4]. Recall from [4, p. 303] that there is a natural pairing, the relative Whitehead product,

$$
\pi_{p}(A) \times \pi_{q}(X, A) \rightarrow \pi_{p+q-1}(X, A), \quad(\alpha, \beta) \rightarrow[\alpha, \beta]
$$


such that

(1) $\partial[\alpha, \beta]=(-1)^{p-1}[\alpha, \partial \beta]$ (the absolute product),

(2) $j_{*}\left[i_{*} \alpha, \beta\right]=\left[\alpha, j_{*} \beta\right]$ where $i_{*}: \pi_{*}(A) \rightarrow \pi_{*}(X)$ and $j_{*}: \pi_{*}(X) \rightarrow$ $\pi_{*}(X, A)$.

2.1 Theorem. Let $Y \in \operatorname{Top}(C), a \in \pi_{p}(Y)$ and $u \in \operatorname{Ker}\left(\hat{y}_{*}\right) \subset \pi_{q}(Y)$. Then $\bar{\Sigma}[a, u]=(-1)^{p-1}\left[\check{y}_{*} \hat{y}_{*} a, \bar{\Sigma} u\right], \bar{\Sigma}[u, a]=-\left[\bar{\Sigma} u, \check{y}_{*} \hat{y}_{*} a\right]$.

Proof. Select $w$ such that $u=\partial_{1} w, k_{*} w=v^{\prime}, \nu v^{\prime}=v=\bar{\Sigma} u$ (see diagram). Let $\bar{w}=[a, w] \in \pi_{p+q}(\bar{K} Y, Y)$. Then

$$
\partial \bar{w}=(-1)^{p-1}[a, w] \text { so } \partial\left((-1)^{p-1} \bar{w}\right)=[a, \partial w]=[a, u] \text {. }
$$

Also $k_{*}\left((-1)^{p-1} \bar{w}\right)=(-1)^{p-1} k_{*}[a, w]=(-1)^{p-1}\left[k_{*} a, k_{*} w\right]$ but $k_{*} a=\hat{y}_{*} a$ so last $=(-1)^{p-1}\left[\hat{y}_{*} a, v^{\prime}\right]$. Thus $\bar{\Sigma}[a, u]=\nu(-1)^{p-1}\left[\hat{y}_{*} a, v^{\prime}\right]$. But $j_{*}\left[\check{y}_{*} \hat{y}_{*} a, \bar{\Sigma} \mu\right]=\left[\hat{y}_{*} a, j_{*} \bar{\Sigma} u\right]$ (use $\check{y}$ for $i$ in (2) above) $=\left[\hat{y}_{*} a, j_{*} v\right]=$ $\left[\hat{y}_{*} a, v^{\prime}\right]$ and $\hat{y}_{*}\left[\check{y}_{*} \hat{y}_{*} a, \bar{\Sigma} u\right]=\left[\hat{y}_{*} a, \hat{y}_{*} \bar{\Sigma} u\right]=0$. Thus

$$
\nu(-1)^{p-1}\left[\hat{y}_{*} a, v^{\prime}\right]=(-1)^{p-1}\left[\check{y}_{*} \hat{y}_{*} a, \bar{\Sigma} u\right]
$$

which proves the first part.

The second part follows from the formula for absolute products $[u, a]=$ $(-1)^{p q}[a, u]$.

2.2 Corollary. Let $Y \in \operatorname{Top}(C), a \in \pi_{p}(Y)$, and $u \in \operatorname{Ker} \hat{y}_{*} \subset \pi_{q}(Y)$. Let $\omega$ be a multiple Whitehead product built up using only a and $u$ such that $u$ appears at least once (e.g. [[[a,u], a], [a,a]]). Then

(1) $\hat{y}_{*} \omega=0$ so $\bar{\Sigma} \omega$ is defined.

(2) If $u$ appears twice then $\bar{\Sigma} \omega=0$.

Proof. (1) $\hat{y}_{*}\left[w^{\prime}, w^{\prime \prime}\right]=\left[\hat{y}_{*} w^{\prime}, \hat{y}_{*} w^{\prime \prime}\right]$ gives (1). (2) Let $w=\left[w^{\prime}, w^{\prime \prime}\right]$ where $w$ has length $n$ and $w^{\prime}, w^{\prime \prime}$ have length $<n$. In Case 1, $u$ appears in both $w^{\prime}$ and $w^{\prime \prime}$. By (1) $\hat{y}_{*} w^{\prime \prime}=0$ so the theorem can be applied yielding $\bar{\Sigma} w=$ $\pm\left[\check{y}_{*} \hat{y}_{*} w^{\prime}, \bar{\Sigma} w^{\prime \prime}\right]$. By (1) $\hat{y}_{*} w^{\prime}=0$ yielding $\bar{\Sigma} w=0$. In Case $2, u$ does not appear in $w^{\prime}$, so $u$ appears at least twice in $w^{\prime \prime}$. By (1) $\hat{y}_{*} w^{\prime \prime}=0$ so 2.1 can be applied. By induction $\bar{\Sigma} w^{\prime \prime}=0$ giving $\bar{\Sigma} w=0$. In Case 3, $u$ does not appear in $w^{\prime \prime}$, result follows as in Case 2.

Our next result is a proof of a theorem of Barcus and Barrat [1]. On the one hand, this illustrates the usefulness of $\bar{\Sigma}$, on the other hand, we will need special cases of the formula later. Before stating the theorem we recall some notations from Hilton [6] and Barcus and Barrat [1]. If $\alpha, \beta \in \pi_{*}(X)$, we have certain (chosen and fixed) basic Whitehead products $p_{k}=p_{k}(\alpha, \beta)$ (e.g., $p_{1}=\alpha, p_{2}=\beta, p_{3}=[\alpha, \beta], p_{4}=[\alpha,[\alpha, \beta]], p_{5}=[\beta,[\alpha, \beta]]$, etc. $)$. Of these, the only ones which contain exactly one $\beta$ are the $\sigma_{k}$ 's, where $\sigma_{k}=\sigma_{k}(\alpha, \beta)$, $\sigma_{-1}=\beta, \sigma_{k}=\left[\alpha, \sigma_{k-1}\right](=[\alpha[\alpha[\cdots[\alpha, \beta] \cdots]])$. Hilton [6] proves 


$$
(\alpha+\beta) \gamma=\sum_{i=-2}^{\infty} p_{i+3} H_{i}(\gamma)
$$

where $H_{i}(\gamma) \in \pi_{*}\left(S^{q}\right), q=q(i), H_{-2}(\gamma)=H_{-1}(\gamma)=\gamma$. The $H_{i}$ 's are homomorphisms and the one corresponding to $\sigma_{k}$ is defined to be $B_{k}$, so all terms $\sigma_{k} B_{k}(\gamma)$ occur in the sum.

2.3 Theorem (BARCUS AND BarRat [1]). $\alpha \in \pi_{m}(X), \beta \in \pi_{n}(X), \gamma \in$ $\pi_{q}\left(S^{m}\right), m, n>1$,

$$
\begin{aligned}
{[\alpha \circ \gamma, \beta] } & =\sum_{j=-1}^{\infty} \varepsilon(j) \sigma_{j+1} \Sigma^{n-1} B_{j}(\gamma), \quad \varepsilon(j)=(-1)^{\delta}, \\
\delta & =n(q+m)+(n+1)(m+1)(j+1) .
\end{aligned}
$$

Proof. It suffices to prove the theorem for $X=S^{m} \vee S^{n}$, and the natural inclusions $\alpha=\iota_{m}, \beta=\iota_{n}$. Two elementary facts are $\iota_{1} \cdot \delta=\left[\iota_{1}, \delta\right]+\delta,\left(\iota_{1} \cdot\right.$ $\left.\iota_{m}\right) \circ \gamma=\iota_{1} \cdot\left(\iota_{m} \circ \gamma\right)$ where "." denotes the action of $\pi_{1}$ on $\pi_{*}$. Applying the first twice and substituting in the second gives $\left(\left[\iota_{1}, \iota_{m}\right]+\iota_{m}\right) \gamma=\left[\iota_{1}, l_{m} \gamma\right]+$ $\iota_{m} \gamma$. Applying the above formula of Hilton gives, after cancellation and skew-commuting,

$$
\left(-1^{q}\right)\left[\iota_{m} \gamma, \iota_{1}\right]=\sum_{i=-1}^{\infty}\left((-1)^{m} p_{i+4}\right) H_{i}(\gamma),
$$

where we define $\bar{p}_{i+4}=p_{i+3}\left(l_{m},\left[l_{m}, \iota_{1}\right]\right)$. If $p_{i+3}=\sigma_{j}$, then $\bar{p}_{i+4}=\sigma_{j+1}\left(l_{m}, l_{1}\right)$. Now apply $\bar{\Sigma}^{n-1}, C=S^{m}$. By 2.2 all $p$ 's except $\sigma$ 's become zero and by 2.1

$$
\bar{\Sigma}^{n-1} \sigma_{j+1}\left(\iota_{m}, \iota_{1}\right)=z \sigma_{j+1}\left(\iota_{m}, \iota_{n}\right) z=(-1)^{\delta}, \quad \delta=(n-1)(j+2)(n-1) .
$$

This gives

$$
(-1)^{q^{\prime}}\left[\iota_{m} \gamma, \iota_{n}\right]=\sum_{j=-1}^{\infty}(-1)^{m} z^{\prime} \sigma_{j+1} B_{j}(\gamma)
$$

where $z^{\prime}=(-1)^{\delta}, \delta=(q-1)(n-1)$. Now $(-1)^{q} z^{\prime}(-1)^{m} z=\varepsilon(j)$ so the theorem follows.

Note on signs. The sign in 2.3 differs from that in [1] but this is due to different sign conventions. For simplicity take $\gamma$ to be a suspension so that 2.3 says $[\alpha \circ \gamma, \beta]=(-1)^{q(n+m)}[\alpha, \beta] \sum^{n-1} \gamma$, or $[\beta, \alpha \circ \gamma]=[\beta, \alpha] \Sigma^{n-1} \gamma$. According to $[16, \mathrm{pp} .7,8]$, a change in orientation will change this formula for 3 reasons: (1) Whitehead product, (2) composition, (3) suspension. If we use the formula in [16, p. 6], $\partial_{n+1}^{\prime}=(-1)^{n} \partial_{n+1}$, and make the corresponding changes in 1, 2 and 3, then the above formula transforms to the Barcus-Barrat version.

Let $(X, A) \in$ Top $(C)$. Define $X / / A=X / R$ where $R$ is the equivalence relation generated by $a \sim \hat{x} a, a \in A, \hat{x} a \in C \subset X$. There is a natural Top (C) $\operatorname{map} k:(X, A) \rightarrow(X / / A, C)$. Define 


$$
T=\partial k_{*}^{-1}: \pi_{i}(X / / A, C) \leftarrow \pi_{i}(X, A) \rightarrow \pi_{i-1}(A, C) .
$$

Note that if $(X, A)=(K Y, Y)$ then $T=\bar{\Sigma}^{-1}$. Assume now that $(X, A)$ is a relative $C W$ complex. Then the homotopy excision theorem implies that if $(X, A)$ is $n$-connected and $(X, C)$ is m-connected then $k_{*}: \pi_{i}(X, A) \rightarrow$ $\pi_{i}(X / / A, C)$ is isomorphic for $i \leqslant m+n$ and epimorphic for $i \leqslant m+n+$ 1. Thus if $i \leqslant m+n, T$ is a genuine homomorphism and if $i=m+n+1$, $T$ is an everywhere defined additive relation. Consider the following sequence

$$
\ldots \rightarrow \pi_{i}(A, C) \stackrel{i_{*}}{\rightarrow} \pi_{i}(X, C) \stackrel{k_{*}}{\rightarrow} \pi_{i}(X / / A, C) \stackrel{T}{\rightarrow} \pi_{i-1}(A, C) \rightarrow .
$$

Each term is acted on by $\pi_{*}(C), c z=[c, z]$. It is a sequence of $\pi_{*}(C)$ modules (or $A\left(\pi_{*}(C)\right.$ )-modules where $A\left(\pi_{*} C\right.$ ) is the universal enveloping algebra) in the sense that $i_{*}$ and $k_{*}$ preserve the action and $T(c z)=$ $(-1)^{q-1} c T(z)$ where $\operatorname{deg} c=q$. The sequence is obtained from the exact sequence of $(X, A, C)$, so it is exact at terms of the form $\pi_{i}(X / / A, C)$, $\pi_{i}(A, C)$ and $\operatorname{Im} i_{*} \subset \operatorname{Ker} k_{*}$ always. The above mentioned conditions on $k_{*}$ prove the following theorem.

2.5 Theorem. Suppose $(X, A) n$-connected, $(A, C)$ m-connected, $N=n+m$ +1 . Then the above sequence is an exact sequence of $\pi_{*}(C)$ modules after the term $\pi_{N}(X, C)$. All T's except possibly the first are homomorphisms and the first is an everywhere defined additive relation.

Now we will consider a special case. Let $Y \in$ Top $(C), X \in$ Top *, $f$ : $X \rightarrow Y \in$ Top (*) such that $\hat{y} f \sim 0$ in Top *. Then we have

$$
\begin{aligned}
& K f \quad \rightarrow \quad K f / / Y \cong C \vee \Sigma X \\
& \uparrow \uparrow \\
& X \stackrel{f}{\rightarrow} \quad Y \stackrel{\hat{y}}{\rightarrow} C
\end{aligned}
$$

$K f \in$ Top $C$ since $\hat{y}$ extends. ( $K f=Y \cup_{f} K X$ is the ordinary mapping cone in Top (*).) Consider the sequence

$$
\begin{aligned}
\pi_{N}(K f, C) \rightarrow \cdots \pi_{i}(Y, C) \stackrel{i_{*}}{\rightarrow} & \pi_{i}(K f, C) \\
& \stackrel{k_{*}}{\rightarrow} \pi_{i}(C \vee \Sigma X, C) \stackrel{T}{\rightarrow} \pi_{i-1}(Y, C) \rightarrow \cdots
\end{aligned}
$$

2.7 TheOREM. $X$ n-connected, $(Y, C)$ m-connected, $N=n+m+2$. Then (2.6) is an exact sequence of $\pi_{*}(C)$-modules. All T's except possibly the first are homomorphisms and the first is an everywhere defined additive relation.

Suppose $X=S^{n_{i}} \vee \cdots \vee S^{n_{w}}, T: \pi_{n_{1}+1}(C \Sigma X, C) \rightarrow \pi_{m_{1}}(Y, C)$. By naturality and examination of the case $X=S^{n}$, and the relation of $T$ to $\Sigma$, one can show that $T\left(l\left(n_{i}+1\right)\right) \ni f_{n_{i}}$. 
3. Primary homotopy operations in Top $(C \rightarrow \mathrm{pt})$.

3.1 Definition. $n, p \geqslant 1, \theta=\left\{\theta_{X}: \pi_{n}(X) \rightarrow \pi_{p}(X)\right\}$ is called an absolute $C$-homotopy operation or a twisted homotopy operation if $f_{*} \theta_{Y}=\theta_{X} f_{*}$ all $f$ : $X \rightarrow Y \in \operatorname{Top}^{C}$.

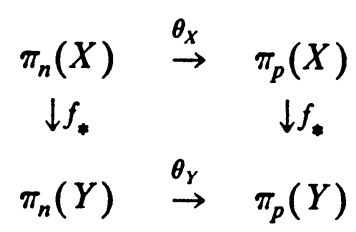

$\mathrm{op}^{\prime}=\mathrm{op}^{\prime}(n, p, C)=$ all such $\theta$.

EXAMPLES. (1) $c \in \pi_{n}(C), \theta_{X}(\alpha)=\left[\check{x}_{*}(c), \alpha\right]$, the Whitehead product. It is a $C$-homotopy operation since $\left(f_{*} \theta_{X}\right)(\alpha)=f_{*}\left[\check{x}_{*} c, \alpha\right]=\left[f_{*} \check{x}_{*} c, f_{*} \alpha\right]$ (naturality of the Whitehead product) $=\left[\check{y}_{*} c, f_{*} \alpha\right]$ (since $f \in$ Top $(C \rightarrow \mathrm{pt})$ ) $=\theta_{Y}\left(f_{*} \alpha\right)=\left(\theta_{Y} f_{*}\right)(\alpha)$.

(2) Any ordinary $(C=p t)$ homotopy operation. More generally if $\theta^{\prime}$ is a $C_{0}$-homotopy operation and $c: C_{0} \rightarrow C$ is a given fixed map then $\theta^{\prime}$ determines a unique $C$-homotopy operations $\theta$ by $\theta_{X}=\theta_{X}^{\prime}$ since any $C$-map is a $C_{0}$-map. Ordinary homotopy operations are pt-homotopy operations and the given map $p t \rightarrow C$ (recall that $C \in$ Top $(p t)$ ) makes them $C$-homotopy operations.

(3) Constant homotopy operations. Let $c \in \pi_{n}(C)$. Define $\theta_{X}(\alpha)=\check{x}_{*}(c)$ for all $\alpha$. Then $\theta$ is a $C$-homotopy operation. These operations are troublesome and we will eliminate them below.

(4) Let $\varepsilon \in \pi_{p}\left(C \vee S^{n}\right)$. Define $\bar{\varepsilon} \in \mathrm{op}^{\prime}(n, p, C)$ by $\bar{\varepsilon}_{X}(\alpha)=\langle\check{x}, \alpha\rangle \varepsilon$.

$$
S^{p} \stackrel{e}{\rightarrow} C \vee S^{n} \stackrel{\langle\dot{x}, \alpha\rangle}{\rightarrow} X
$$

(5) $\theta, \theta^{\prime} \in \mathrm{op}^{\prime}, \theta+\theta^{\prime} \in \mathrm{op}^{\prime}$ if $\left(\theta+\theta^{\prime}\right)_{X}(\alpha)=\theta_{X}(\alpha)+\theta_{X}^{\prime}(\alpha)$. In fact op' is a group, abelian if $p \geqslant 2$. The constant operations form a subgroup.

3.2 Defintion. op $=\mathrm{op}(n, p, C)=\operatorname{ker}(\zeta)$ where $\zeta: \mathrm{op}^{\prime}(n, p, c) \rightarrow \pi_{p}(C)$ is the homomorphism defined by $\zeta(\theta)=\theta_{C}(0)$.

We can also define $\sigma: \pi_{p}(C) \rightarrow$ op' by $\sigma(c)_{X}(x)=\check{x}_{*}(c)$, all $X$, all $x$. Then $\zeta \sigma=$ id and if $p>2$ then op' is the direct sum of op and $\pi_{p}(C)$. We wish to have other representations of op.

(3.2b) $\mathrm{op}_{1}=\mathrm{op}_{1}(n, p, C)=$ all $\theta, \theta=\left\{\theta_{X}: \pi_{n}(X) \rightarrow \pi_{p}(X, C)\right\}$ such that $f_{*} \theta_{X}=\theta_{Y} f_{*}$ for $f \in$ Top $^{C}$. (Here $\pi_{p}(X, C)$ is $\pi_{p}(\tilde{X}, C)$ where $\tilde{X}$ is the mapping cylinder of $\check{x}_{\text {.) }}$

(3.2c) $\mathrm{op}_{2}=\mathrm{op}_{2}(n, p, C)=$ all $\theta, \theta_{X}: \pi_{n}(X, C) \rightarrow \pi_{p}(X, C)$ for $X \in$ Top $(C)$ and $f_{*} \theta_{X}=\theta_{Y} f_{*}$ for $X \in$ Top $(C)$.

(3.2d) $\mathrm{op}_{3}=\mathrm{op}_{3}(n, p, C)=\mathrm{op}^{\prime}(n, p, C) / R$ where $R$ is the equivalence 
relation defined by $\theta R \theta^{\prime}$ if $\theta$ and $\theta^{\prime}$ differ by a constant operation (so $o p_{3}$ is the quotient group if $p \geqslant 2$ ).

Let $\theta \in$ op. Define $\theta_{1} \in$ op $p_{1}$ by $\theta_{1}=j_{*}(\theta), j_{*}: \pi_{p}(X) \rightarrow \pi_{p}(X, C)$. For $\theta_{1} \in \mathrm{op}_{1}$ define $\theta_{2} \in \mathrm{op}_{2}$ by $\theta_{2}=\theta_{1} \nu$

$$
0 \rightarrow \pi_{i}(C) \underset{\dot{x}_{*}}{\stackrel{\hat{x}_{*}}{\leftrightarrows}} \pi_{i}(X) \underset{\dot{j}_{*}}{\stackrel{\nu}{\leftrightarrows}} \pi_{i}(X, C) \rightarrow 0 \quad \nu=\nu_{X}, X \in \operatorname{Top}(C) .
$$

Let $X \in$ Top $^{c}$. Define $\bar{X} \in$ Top $(C)$ and $u: \bar{X} \rightarrow X \in$ Top $^{c}$ by $\bar{X}=C \vee$ $X, i_{1}: C \rightarrow C \vee X, \pi_{1}: C \vee X \rightarrow C . u=\langle x$, id $\rangle$. We also have $i=i_{2}$ : $X \rightarrow \bar{X} \in \operatorname{Top}(*), u i=$ id, $u_{*}$ sends $\operatorname{Ker} i_{*}$ onto $\pi_{n} X$. Also $f: X \rightarrow Y \in \operatorname{Top}^{c}$ gives

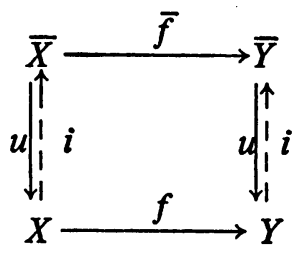

where $\bar{f}=1 \bigvee f$.

Now let $\Gamma \in$ op $_{2}$. Define $\Gamma^{\prime}$ by $\Gamma^{\prime}=u_{*} \nu \Gamma_{\bar{X}} j_{*} i_{*}$. The comments above on $\bar{X}$ show that $\Gamma^{\prime}$ is in op. Finally, define op $\rightarrow o p_{3}$ by op $\subset \mathrm{op}^{\prime} \rightarrow o p_{3}$.

3.3 THEOREM. $\mathrm{op} \cong \mathrm{op} \mathrm{p}_{1} \cong o \mathrm{p}_{2} \cong o \mathrm{p}_{3}$.

Proof. It is not hard to check that the above defined maps are isomorphisms.

We will work with $\operatorname{Top}^{2}(C \rightarrow \mathrm{pt})=$ all tuples $\left(X, X_{0}, \check{x}\right)$ with $\check{x}: C \rightarrow X_{0}$, but we will write $\left(X, X_{0}\right) \in$ Top $^{C}$.

3.4 Definition. rel - op $=\operatorname{rel}$ - op $(n, p, C)=$ all $\theta, \theta=\left\{\theta_{X, X_{0}}: \pi_{n}\left(X, X_{0}\right) \rightarrow\right.$ $\left.\pi_{p}\left(X, X_{0}\right)\right\}$ where $f_{*} \theta_{\left(X, X_{0}\right)}=\theta_{\left(Y, Y_{0}\right)} f_{*}$ for $f:\left(X, X_{0}\right) \rightarrow\left(Y, Y_{0}\right) \in$ Top ${ }^{C}$.

Note that $\theta \in$ rel - op implies $\theta 0=0$ since $\check{x}:(C, C) \rightarrow\left(X, X_{0}\right) \in$ Top $^{C}$ and $\theta 0=\theta \check{x}_{*} 0=\check{x}_{*} \theta 0=\check{x}_{*} 0=0$. We will define three functions:

$$
\begin{aligned}
& \text { rel - op }(n, p, C) \stackrel{r}{\rightarrow} \mathrm{op}(n, p, C), \\
& \text { rel - op }(n, p, C) \stackrel{b}{\rightarrow} \mathrm{op}(n-1, p-1, C), \quad b \text { bijection, } \\
& \mathrm{op}(n, p, C) \stackrel{s}{\rightarrow} \mathrm{op}(n+1, p+1, C), \quad s=r b^{-1} .
\end{aligned}
$$

First rel $-\mathrm{op}(n, p, C) \rightarrow^{r_{1}} \mathrm{op}_{2}(n, p, C) \cong \mathrm{op}(n, p, C)$ where the map is $\left(r_{1} \theta\right)_{X}$ $=\theta_{X, C}$ for $X \in \operatorname{Top}(C)$ so $(r \theta)_{X}=u_{*} \nu \theta_{\bar{X}, C} j_{*} i_{*}$. The following theorem is not difficult.

3.5 THEOREM. (a) $\left(X, X_{0}\right) \in$ Top $^{c}$. Then the following diagram is commutative 


$$
\begin{array}{ccc}
\pi_{n}(X) & j_{*} & \\
\downarrow \text { 时 } & & \pi_{n}\left(X, X_{0}\right) \\
& j_{*} & \downarrow \theta \\
\pi_{p}(X) & \rightarrow & \pi_{p}\left(X, X_{0}\right)
\end{array}
$$

(b) $r \theta$ is characterized by the property in (a).

Now let $\theta \in$ rel - op $(n, p, C)$. Define $(b \theta)_{X}=\partial \theta_{K X, X} \partial^{-1}$ for $X \in$ Top $^{C}$

$$
\pi_{n-1}(X) \stackrel{\partial}{\leftarrow} \pi_{n}(K X, X) \stackrel{\theta}{\rightarrow} \pi_{p}(K X, X) \stackrel{\partial}{\rightarrow} \pi_{p-1}(X)
$$

$X \in \operatorname{Top}^{c}$ so $(K X, X) \in \operatorname{Top}^{c} . b \theta$ is clearly natural and $\theta 0=0$ implies $b \theta$ is in $\operatorname{ker}(\zeta)=$ op.

3.6 THEOREM. (a) $\left(X, X_{0}\right) \in$ Top $^{C}$. Then the following diagram is commutative

$$
\begin{array}{ccc}
\pi_{n}\left(X, X_{0}\right) & \stackrel{\partial}{\rightarrow} & \pi_{n-1}\left(X_{0}\right) \\
\downarrow & & \downarrow b \theta \\
\pi_{p}\left(X, X_{0}\right) & \stackrel{\partial}{\rightarrow} & \pi_{p-1}\left(X_{0}\right)
\end{array}
$$

(b) $b \theta$ is characterized by the property in (a).

Proof. (b) is clear. For (a) note first that if $Y \in \operatorname{Top}(C)$ we have

$$
\begin{aligned}
& \pi_{n-1}(Y) \leftarrow \pi_{n}(K Y, Y) \rightarrow \pi_{p}(K Y, Y) \rightarrow \pi_{p-1}(Y) \\
& \|\quad \uparrow \quad \uparrow \quad\| \\
& \pi_{n-1}(Y) \leftarrow \pi_{n}(\bar{K} Y, Y) \rightarrow \pi_{p}(\bar{K} Y, Y) \rightarrow \pi_{p-1}(\bar{K} Y)
\end{aligned}
$$

so the result is true for $\left(X, X_{0}\right)=(\bar{K} Y, Y)$ any $Y \in$ Top $C$. But if $x \in$ $\pi_{n}\left(X, X_{0}\right)$ we can find $Y \in$ Top $C$,

$$
u:(\bar{K} Y, Y) \rightarrow\left(X, X_{0}\right) \in \operatorname{Top}^{c}, \quad y \in \pi_{n}(\bar{K} Y, Y), u_{*} y=x
$$

specifically $(\bar{K} Y, Y)=\left(C \vee K S^{n-1}, C \vee S^{n-1}\right), u=\langle\check{x}, \bar{x}\rangle$ work where $\bar{x}$ represents $x$. Then $(b \theta) \partial x=(b \theta) \partial u_{*} y=u_{*}(b \theta)_{Y} \partial y=u_{*} \partial \theta_{(\bar{K} Y, Y)} y$ $=\partial \theta_{\left(x, x_{0}\right)} u_{*} y=\partial \theta x$.

Let $\left(X, X_{0}\right) \in$ Top $^{c}$. Define $Q=Q\left(X, X_{0}\right)$ by $Q=\left\{w \in X^{I} \mid w(0) \in\right.$ $\left.\check{x}(C), w(1) \in X_{0}\right\}$. Then $Q \rightarrow C, w \rightarrow w(0)$ is a Top * fibration with fiber

$$
P=P\left(X, X_{0}\right)=\left\{w \in X^{I} \mid w(0)=*, w(1) \in X_{0}\right\}
$$

and cross section

$$
\sigma: C \rightarrow Q, \sigma(c)=c^{I}=\text { constant path at } c .
$$

The correspondence $\left(X, X_{0}\right) \rightarrow(P \rightarrow Q \leftrightharpoons C)$ is functorial. It is well known that adjointness gives $\pi_{n}\left(X, X_{0}\right) \cong \pi_{n-1}(P)$ and that this is natural. Here we also have 


$$
\begin{aligned}
& 0 \rightarrow \pi_{*}(P) \stackrel{i}{\rightarrow} \pi_{*}(Q) \stackrel{\rightarrow}{\rightarrow} \pi_{*}(C) \quad \rightarrow 0 \\
& 0 \rightarrow \pi_{*}(C) \stackrel{j_{*}}{\leftrightarrows} \pi_{*}(Q) \stackrel{\rightarrow}{\rightarrow} \pi_{*}(Q, C) \rightarrow 0 .
\end{aligned}
$$

Thus $j_{*} j_{*}: \pi_{k}(P) \rightarrow \pi_{k}(Q, C)$ is an isomorphism, natural in $\left(X, X_{0}\right)$. So, $\pi_{n}\left(X, X_{0}\right) \cong \pi_{n-1}(Q, C)$ and this is natural also.

Now define $c:$ op $(n-1, p-1, C)=\mathrm{op}_{2}(n-1, p-1, C) \rightarrow$ rel - op $(n, p, C)$ by $c(\theta)=\Gamma$

$$
\pi_{n}(X, \underbrace{\left.X_{0}\right) \approx \pi_{n-1}(Q, C) \stackrel{\theta_{Q}}{\rightarrow} \pi_{p-1}(Q, C) \approx \pi_{p}}_{\Gamma_{X, X_{0}}}\left(X, X_{0}\right) .
$$

3.7 THEOREM. $c=b^{-1}$.

Proof. This is most easily proven by using Theorem 3.10 below.

We can now define $s=r b^{-1}: \mathrm{op}(n, p, C) \rightarrow \mathrm{op}(n+1, p+1, C)$,

3.8 ThEOREM. (a) Let $X \in \operatorname{Top}(C)$. Then the following diagram is commutative

$$
\begin{array}{ccc}
\pi_{n}(X, C) & \stackrel{\bar{\Sigma}}{\rightarrow} & \pi_{n+1}(\bar{\Sigma} X, C) \\
\downarrow \theta & & \downarrow\lrcorner \theta \\
\pi_{p}(X, C) & \stackrel{\bar{\Sigma}}{\rightarrow} & \pi_{p+1}(\bar{\Sigma} X, C)
\end{array}
$$

(b) $s \theta$ is characterized by the property in (a).

3.9 Definition.

$$
\begin{aligned}
& \nu^{\prime}: \pi_{p}\left(C \vee S^{n}\right) \rightarrow \mathrm{op}^{\prime}(n, p, C), \\
& \nu: \pi_{p}\left(C \vee S^{n}, C\right) \rightarrow \mathrm{op}(n, p, C), \\
& \nu: \pi_{p}\left(C \vee E^{n}, C \vee S^{n-1}\right) \rightarrow \operatorname{rel}-\mathrm{op}(n, p, C) .
\end{aligned}
$$

$\left(\nu^{\prime} \varepsilon\right)_{X}(\alpha)=\langle\check{x}, \alpha\rangle \varepsilon$. Let $\pi: C \vee S^{n} \rightarrow C$ be the projection, $\nu^{\prime}\left(\operatorname{Ker} \pi_{*}\right) \subset$ $\operatorname{Ker} \zeta$ so $\nu$ defined by $\pi_{p}\left(C \vee S^{n}, C\right) \cong \operatorname{ker} \pi_{*} \rightarrow^{v^{\prime}} \operatorname{Ker} \zeta=$ op. $(\overline{\nu \varepsilon})_{\left(X, x_{\alpha}\right)}(\alpha)$ $=\langle\check{x}, \alpha\rangle \varepsilon$. Note that if $\check{x}: C \rightarrow X_{0}$ and $w:\left(A, A_{0}\right) \rightarrow\left(X, X_{0}\right)$, then

$$
\langle\check{x}, w\rangle:\left(C \vee A, C \vee A_{0}\right) \rightarrow\left(X, X_{0}\right) \text {. }
$$

3.10 THEOREM. (a) $\nu^{\prime}, \nu, \bar{\nu}$ are bijections and isomorphisms when the sets are groups.

(b) $\nu^{\prime-1}(\theta)=\theta_{C \vee s^{n}}\left(\iota_{n}\right), \nu^{-1}(\theta)=\theta_{\left(C \vee S^{n}, C\right)}\left(\mu_{n}\right)$ where $\theta \in \mathrm{op}_{2}, \bar{\nu}^{-1}(\theta)=$ $\theta_{C \vee E^{n}, C \vee S^{n-1}}\left(\varepsilon_{n}\right)$.

Proof. Simply adapt the usual categorical proof about natural transformations of representable functors, e.g., B. Mitchel, Theory of categories, (p. 97, Lemma 2.1) to the present setting. 
3.11 THEOREM. Under the bijections of 3.9 the functions $r, b, s$, correspond to

$$
\begin{aligned}
& \mu_{*}: \pi_{p}\left(C \vee E^{n}, C \vee S^{n-1}\right) \rightarrow \pi_{p}\left(C \vee S^{n}, C\right), \\
& \partial: \pi_{p}\left(C \vee E^{n}, C \vee S^{n-1}\right) \rightarrow \pi_{p-1}\left(C \vee S^{n-1}, C\right) \text { bijection, } \\
& \mu_{*} \partial^{-1}=\bar{\Sigma}: \pi_{p}\left(C \vee S^{n}, C\right) \rightarrow \pi_{p+1}\left(C \vee S^{n+1}, C\right) .
\end{aligned}
$$

Proof. Not difficult using 3.5 and 3.6.

\section{Stable primary operations.}

4.1 Defintion. (a) A stable $C$-homotopy operation of degree $q$ is a family $\theta=\left\{\theta^{n}\right\}$ where

(1) $q+2 \leqslant n<\infty$,

(2) $\theta^{n} \in \mathrm{op}(n, n+q, C)$,

(3) $s \theta^{n}=\theta^{n+1}$.

(b) $G_{q}(C)=$ all stable $C$-homotopy operations of degree $q$.

Note that by 1.10 and $3.11, G_{q}(C) \cong \pi_{2 q+2}\left(C \vee S^{q+2}, C\right) \cong \pi_{n+q}(C \vee$ $\left.S^{n}, C\right)$ for any $n \geqslant q+2$. There is a composition operation $G_{q} \times G_{k} \rightarrow G_{k+q}$ defined by $\theta \theta^{\prime}=\Gamma$ where $\Gamma^{n}=\theta^{n+k} \theta^{\prime n}: \pi_{n} \rightarrow \pi_{n+q+k}$. They need only be defined for $n>q+k+2$ and then $\theta^{\prime n}$ and $\theta^{n+k}$ are defined. $G$ is clearly an associative graded ring.

Notice that stable homotopy operations act on more than just stable homotopy. In fact for $X \in$ Top $^{C}$ we have

$$
G_{q}(C) \times \pi_{n}(X) \rightarrow \pi_{n+q}(X) \text { all } X \text {, all } n \geqslant q+2 .
$$

For example (1) $c \in \pi_{q+1}(C), \theta^{n} x=(-1)^{n q}[c, x], x \in \pi_{n}(X), n>q+2$. Then $\theta \in G_{q}(C)$. (2) $c \in \pi_{2}(C), \eta_{n} \in \pi_{n+1}\left(S^{n}\right), n>3, \Sigma \eta_{n}=\eta_{n+1} . \theta^{n} x=$ $(-1)^{n}[c, x]+\eta_{n} x$. Then $\theta \in G_{1}(C)$.

From $\$ 3$ we see that $s \theta^{n}=\theta^{n+1}$ iff $r b^{-1} \theta^{n}=\theta^{n+1}$. So for $n>q+3, \theta^{n}$ is the restriction of a uniquely determined relative homotopy operation. So we have for $(X, A) \in \operatorname{Top}^{c}$

$$
G_{q}(C) \times \pi_{n}(X, A) \rightarrow \pi_{n+q}(X, A)
$$

all $n>q+3$ and a commutative ladder:

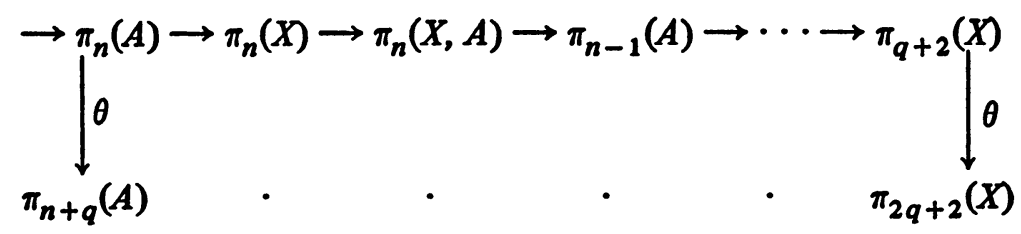

The ladder can be extended but uniqueness is lost.

By 3.8, condition (3) is equivalent to: for all $X \in$ Top $(C)$ the following diagram is commutative: 


$$
\begin{array}{ccc}
\pi_{n}(X, C) & \stackrel{\theta \pi}{\rightarrow} & \pi_{n+q}(X, C) \\
\downarrow \bar{\Sigma} & & \downarrow \bar{\Sigma} \\
\pi_{n+1}(\bar{\Sigma} X, C) & \stackrel{\theta \frac{n+1}{\Sigma} X}{\rightarrow} & \pi_{n+1+q}(\bar{\Sigma} X, C)
\end{array}
$$

Let $\beta_{j} \in G(C), \operatorname{deg} \beta_{j}=b_{j}, 1<j \leqslant w$. Write

$$
\beta^{n}=\left(\beta_{1}^{n}, \ldots, \beta_{w}^{n}\right): \pi_{n}(X) \rightarrow \bigoplus_{j=1}^{w} \pi_{n+b_{j}}(X), \quad X \in \operatorname{Top}^{c} .
$$

Let $J^{n+b}=S^{n+b_{1}} \vee \cdots \vee S^{n+b_{w}}$ so $\beta^{n}: J^{n+b} \rightarrow C \vee S^{n}$ as a map. The following three results are special cases of results of Kruse [7], [8, Corollary 2.10, 2.12a]

4.2. The following sequence is an exact sequence of abelian groups.

$$
\oplus \pi_{n+b_{j}}(X) \stackrel{\beta^{n}}{\leftarrow} \pi_{n}(X) \stackrel{b^{*}}{\leftarrow}\left[K\left(\beta^{n}\right), X\right] \stackrel{c \bar{k}}{\leftarrow} \oplus \pi_{n+b_{j}+1} X^{\beta^{n+1}} \pi_{n+1}(X)
$$

where $S^{n} \subset C \vee S^{n} \subset K(\beta)$ is $b$ and $\bar{k}$ is defined by $\bar{k}(f)=(\check{x}, f) k_{1}$

$$
\begin{array}{lll}
K \beta & \stackrel{k_{1}}{\rightarrow} & P \simeq C \vee \Sigma J \\
\uparrow & & \uparrow \\
C \vee S^{n} & \stackrel{\pi_{1}}{\rightarrow} & C
\end{array}
$$

4.3. There is a homotopy equivalence in Top ${ }^{c}, a: K\left(\beta^{n}\right) \rightarrow \bar{\Sigma} K\left(\beta^{n-1}\right)$, giving a commutative diagram

$$
\begin{array}{ccccc}
C \vee S^{n} & \stackrel{i}{\rightarrow} & K\left(\beta^{n}\right) & \stackrel{k}{\rightarrow} & \bar{\Sigma}^{2}\left(C \vee J^{n+b-1}\right) \\
\| & & \downarrow a & & \downarrow t \\
\bar{\Sigma}\left(C \vee S^{n-1}\right) & \stackrel{i}{\rightarrow} & \bar{\Sigma} K\left(\beta^{n-1}\right) & \stackrel{k}{\rightarrow} & \bar{\Sigma}^{2}\left(C \vee J^{n+b-1}\right)
\end{array}
$$

where $t$ interchanges the suspension variables. Here $C \rightarrow K \beta$ comes from $C \vee S^{n} \subset K \beta$ and $K \beta \rightarrow C$ because $\pi_{1} \beta \sim 0$.

4.4. Let $g: J^{n}=S^{n_{1}} \vee \cdots \vee S^{n_{w}} \rightarrow Y, Y \in$ Top $(C), \hat{y} g \sim 0$, so $\bar{\Sigma} g$ : $J^{n+1} \rightarrow \bar{\Sigma} Y$. Then for $Z \in$ Top $^{C}$

$$
[\bar{\Sigma} Y, Z] \stackrel{\left(\bar{\Sigma}_{g}\right)^{*}}{\rightarrow} \oplus \pi_{n(i)}(Z) .
$$

$(\bar{\Sigma} g)^{*}$ is a homomorphism.

5. Stable secondary operations. Let $\alpha_{j}, \beta_{j} \in G(C), \operatorname{deg} \alpha_{j}=a_{j}, \operatorname{deg} \beta_{j}=b_{j}$, $a_{j}+b_{j}=k, 1 \leqslant j<w$. Assume

$$
\sum_{j=1}^{w} \alpha_{j} \beta_{j}=0 \text { in } G_{k}(C) \text {. }
$$

Let $\beta=\left(\beta_{1}, \ldots, \beta_{w}\right): \pi_{n}(X) \rightarrow \bigoplus_{i=1}^{w} \pi_{n+b}(X), \quad X \in \operatorname{Top}^{c}, \alpha=$ $\left\langle\alpha_{1}, \ldots, \alpha_{w}\right\rangle: \bigoplus \pi_{n+b_{j}}(X) \rightarrow \pi_{n+k}(X)$ and write 5.1 as $\alpha \beta=0$. 
We will associate to 5.1 an operation $\Phi=\left\{\Phi^{n}\right\}$ such that

(1) $k+3 \leqslant n<\infty$.

(2) $\Phi^{n}(X): \pi_{n}(X) \rightarrow \pi_{n+k+1}(X)$ is an additive relation, natural for $X \in$ Top ${ }^{C}$ [i.e. $f: X \rightarrow Y, x \in \operatorname{Dom} \Phi(X) \Rightarrow f_{*} x \in \operatorname{Dom} \Phi(Y)$ and $f_{*} \Phi(x) \subset$ $\left.\Phi f_{*}(x)\right]$ and such that

$$
\operatorname{domain}(\Phi)=\operatorname{Ker}(\beta) \subset \pi_{n}(X)\left[\operatorname{Ker}(\beta)=\cap \operatorname{Ker}\left(\beta_{j}\right)\right],
$$

indeterminacy $\Phi=\operatorname{Im} \alpha \subset \pi_{n+k+1}(X)\left[\operatorname{Im} \alpha=+\operatorname{Im} \alpha_{j}^{u}, u=n+b_{j}+1\right]$.

(3) $X \in \operatorname{Top}(C=C)$, then $\bar{\Sigma} \Phi \subset(-\Phi \Sigma)$ on ker $\hat{x}_{*} \subset \pi_{n}(X)$.

(4) $(X, A) \in \operatorname{Top}^{C}, \pi_{n}(A) \rightarrow^{i} \cdot \pi_{n}(X) \rightarrow^{j} \cdot \pi_{n}(X, A) \rightarrow^{\partial} \pi_{n-1}(A) \rightarrow \pi_{n-1}(X)$, $n \geqslant k+3, x \in \operatorname{Dom} \Phi \subset \pi_{n}(X), j_{*} x=0$. Then $j_{*} \Phi x=\alpha \partial^{-1} \beta i_{*}^{-1} x$.

5.2 THEOREM. (a) There is an operation satisfying (1)-(4).

(b) If $\Phi$ and $\Phi^{\prime}$ both satisfy (1)-(4) then there is a primary operation $\gamma \in G_{k+1}(c)$ such that $\Phi^{\prime n}=\Phi^{n}+(-1)^{n} \gamma^{n}$.

(c) Let $i=\left(-, b_{n}\right): C \vee S^{n} \subset K\left(\beta^{n}\right)$ so $b_{n} \in \operatorname{Ker}(\beta) \subset \pi_{n}\left(K \beta^{n}\right)$. Suppose $\Phi$ and $\Phi^{\prime}$ satisfy (1)-(4) and for some $n_{0}, k+3<n_{0}, \Phi(b)=\Phi^{\prime}(b), b=b_{n_{0}}$. Then $\Phi=\Phi^{\prime}$.

Proof. We first construct $\Phi$. We have $\beta_{j}: S^{n+b_{j}} \rightarrow C \vee S^{n}$, using $\beta_{j} \in$ $\pi_{*}\left(C \vee S^{n}, C\right) \cong \operatorname{ker} \pi_{*} \subset \pi_{*}\left(C \vee S^{n}\right)$. Set $J^{n}=S^{n+b_{1}} \vee \cdots \vee S^{n+b_{w}}$ so that $\beta: J^{n} \rightarrow C \vee S^{n}$. The $\alpha_{j}$ 's give

$$
\alpha \in \pi_{n+k}\left(C \vee J^{n}, C\right), \quad \alpha=\Sigma \alpha_{j}\left(\left(\left(n+b_{j}\right)\right)\right. \text {. }
$$

The sequence $J^{n} \rightarrow C \vee S^{n} \rightarrow K \beta$ gives an exact sequence (2.6)

$$
\begin{aligned}
\pi_{N}\left(C \vee S^{n}, C\right) & \rightarrow \cdots \rightarrow \pi_{j+1}(C \vee \Sigma J, C) \stackrel{T}{\rightarrow} \pi_{j}\left(C \vee S^{n}, C\right) \\
& \rightarrow \pi_{j}(K \beta, C) \rightarrow \pi_{j}(C \vee \Sigma J, C) \rightarrow \ldots
\end{aligned}
$$

where $N=2 n+b, b=\min \left\{b_{j}\right\}$. We need the portion

$$
\begin{aligned}
\pi_{n+k+1}\left(C \vee S^{n}, C\right) & \rightarrow \pi_{n+k+1}(K \beta, C) \stackrel{k}{\rightarrow} \pi_{n+k+1}(C \vee \Sigma J, C) \\
& \stackrel{T}{\rightarrow} \pi_{n+k}\left(C \vee S^{n}, C\right)
\end{aligned}
$$

which is exact since $n \geqslant k+2$ gives $n+k+1<n+n-1<N$ (so $T$ is a homomorphism). We compute

$$
T(\Sigma \alpha)=T \alpha\left(\Sigma l\left(n+b_{j}+1\right)\right)=\alpha T()=\alpha\left(\Sigma \beta_{j}\right)=\alpha \beta=0 .
$$

So there is an $\tilde{\alpha} \in \pi_{n+k+1}(K \beta, C)$ with $k_{*} \tilde{\alpha}=\Sigma \alpha$. We can view $\tilde{\alpha}$ as a map $S^{n+k+1} \rightarrow K \beta$ since $\pi_{j}(K \beta, C) \cong \operatorname{ker} \hat{k}_{*} \subset \pi_{j}(K \beta)$. We want to make the choice of $\tilde{\alpha}$ a little more specific. For $n=k+2$ choose $\tilde{\alpha}=\tilde{\alpha}(k+2)$ such that $k_{*} \tilde{\alpha}=\Sigma \alpha$. Now let $a: K\left(\bar{\Sigma}^{n+1} \beta\right) \rightarrow \bar{\Sigma} K\left(\bar{\Sigma}^{n} \beta\right)$ be the homotopy equivalence of 4.3. Define $\tilde{\alpha}^{n+1}=-a_{*}^{-1} \bar{\Sigma} \tilde{\alpha}^{n}$. Then, from 4.3, we see that $k_{*} \tilde{\alpha}^{n}=$ 
$\bar{\Sigma} \alpha$ for all $n$. Also for $n \geqslant k+3, \tilde{\alpha}^{n}$ is a $\bar{\Sigma}$ element so by 4.4 gives a homomorphism in homotopy.

Consider now, for $n>k+3$

$$
\pi_{n}(X) \stackrel{b^{*}}{\leftarrow}[K \beta, X]^{c \stackrel{\tilde{\alpha}^{*}}{\rightarrow}} \pi_{n+k+1}(X)
$$

and define $\Phi_{X}^{n}=\tilde{\alpha}^{*} b^{*-1}: \pi_{n}(X) \rightarrow \pi_{n+k+1}(X)$. Say that $S^{n+k+1} \rightarrow K(\beta) \leftarrow$ $S^{n}$ represents $\Phi . \Phi^{n}$ is clearly natural on Top ${ }^{c}$ and an additive relation since $i^{*}$ and $\tilde{\alpha}^{*}$ are homomorphisms. The exact sequence 4.2 shows that Dom $\Phi=$ $\operatorname{im} b^{*}=\operatorname{ker} \beta$. From the same sequence we have

$$
\text { Ind } \Phi=\tilde{\alpha}^{*}\left(\operatorname{Ker} b^{*}\right)=\tilde{\alpha}^{*}\left(\operatorname{Im} k^{*}\right)=\operatorname{Im}\left(\tilde{\alpha}^{*} k^{*}\right)=\operatorname{Im}(\Sigma \alpha)^{*}=\operatorname{Im} \alpha
$$

and this completes the proof of (2). (1) is by definition. Let $x \in \operatorname{ker} \hat{x}_{*} \cap$ Dom $\Phi \subset \pi_{n}(X)$. Then $\beta x=0$ implies $\beta \bar{\Sigma} x=\bar{\Sigma} \beta x=0$ so $\bar{\Sigma} x \in \operatorname{Dom} \Phi_{\bar{\Sigma} x}$. It is not hard to show that $y \in \bar{\Sigma} \Phi x \Rightarrow y \in-\Phi \bar{\Sigma} x$ proving (3).

Now let $\Psi=\alpha \partial^{-1} \beta i_{*}^{-1}$. The diagram for $\Psi$ as follows:

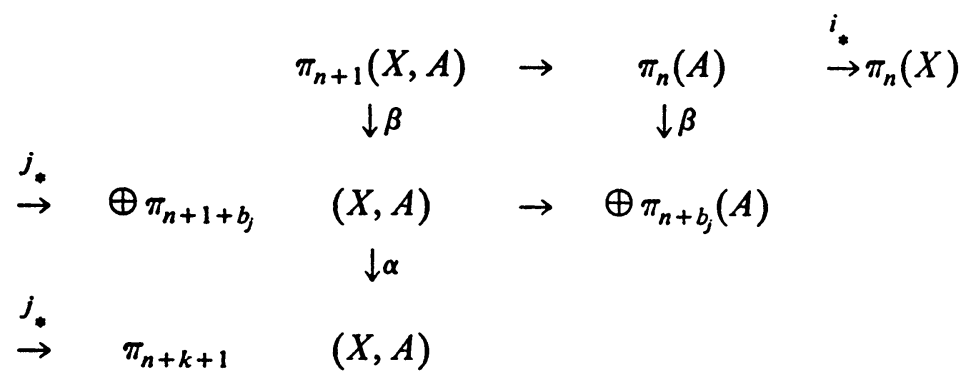

The indeterminacy from $i_{*}^{-1}$ is killed by the final $\alpha$ so $\operatorname{Ind} \Psi=\operatorname{Im} \alpha j_{*}=$ $\operatorname{Im}\left(j_{*} \alpha\right)=\operatorname{Ind} j_{*} \Phi$ and to prove (4) it will suffice to find a common element.

First take $(X, A)=\left(K \beta, C \vee S^{n}\right), \quad x=b: S^{n} \rightarrow K \beta$. Now $\bar{k}_{*}$ : $\pi_{n+k+1}\left(K \beta, C \vee S^{n}\right) \rightarrow \pi_{n+k+1}(C \vee \Sigma J, C)$ is isomorphic so it suffices to find a common element of $\bar{k}_{*} j_{*} \Phi(b)$ and $\bar{k}_{*} \Psi(b)$. Note $\tilde{\alpha} \in \Phi(b)$ so $\bar{\Sigma} \alpha \in$ $\bar{k}_{*} j_{*} \Phi(b)=k_{*} \Phi(b)$. Also

$$
\begin{aligned}
\bar{k}_{*} \alpha \partial^{-1} \beta i_{*}^{-1}(b) \supset \bar{k}_{*} \alpha \partial^{-1} \beta_{n} & =\alpha \bar{k}_{*} \partial^{-1} \beta=\alpha T^{-1} \beta \\
& \ni \alpha\left(\bigoplus \iota\left(n+b_{j}+1\right)\right)=\bar{\Sigma} \alpha
\end{aligned}
$$

which proves the result for this case. The general case follows from a naturality argument. This completes the proof of (a).

Let $\Phi$ and $\Phi^{\prime}$ both satisfy (1)-(4). Let $n=k+3, \tilde{\alpha} \in \Phi(b)$ and $\tilde{\alpha}^{\prime} \in \Phi^{\prime}(b)$. From (4) we see that $k_{*} \tilde{\alpha}=\Sigma \alpha=k_{*} \tilde{\alpha}^{\prime}$ so $\tilde{\alpha}^{\prime}-\tilde{\alpha}=b_{*} \gamma^{\prime}=b_{*}(-1)^{n} \gamma$ in 2.6. Let $\tilde{x}: k \beta \rightarrow X, \tilde{x} i=(\tilde{x}, x)\left(i=(-, b): C \vee S^{n} \rightarrow K \beta\right)$. Then $\tilde{x} \alpha^{\prime} \in \tilde{x}_{*} \Phi^{\prime} i \subset$ $\Phi^{\prime} x$ and $\tilde{x}_{*} \alpha+\tilde{x}_{*} b_{*}(-1)^{n} \gamma \in \tilde{x}_{*} \Phi b+x_{*}(-1)^{n} \gamma \subset \Phi x+(-1)^{n} \gamma x$.

So $\Phi^{\prime} x$ and $\Phi x+(-1)^{n} \gamma x$ have a common element, so are equal. Axiom 3 shows that if $(-1)^{n} \gamma$ works for $\Phi^{n}$ and $\Phi^{\prime n}$ then $(-1)^{n+1} \gamma$ will work for $\Phi^{n+1}$ 
and $\Phi^{\prime n+1}$. This proves (b) and the same argument proves (c).

We consider two examples.

Example 1. Let $C=Q P^{2}, c: S^{4}=Q P^{1} \subset Q P^{2}$, so $c \in \pi_{4}\left(Q P^{2}\right)$. Recall that $Q P^{2}=S^{4} \cup_{\gamma} e^{8}, \gamma=\gamma_{4}: S^{7} \rightarrow S^{4}$ the Hopf map, so $c \gamma=0$ in $\pi_{7}\left(Q P^{2}\right)$.

Define $\alpha_{m} \in G_{3}(C)$ by $\alpha_{m}=\left\{\alpha_{m}^{j}\right\}$ and $\alpha_{m}^{j}: \pi_{j}(Z) \rightarrow \pi_{j+3}(Z), Z \in$ Top $^{C}$, $j \geqslant 4, \alpha_{m}^{j}(z)=m z \gamma+(-1)^{j}[\bar{c}, z]$. Here $\gamma=\gamma_{j}=\Sigma^{j-3} \gamma_{4}$ and $\bar{c}=\check{z}_{*} c \in$ $\pi_{4}(Z)$. We claim that there is a relation

$$
\alpha_{m+1} \alpha_{m}=0 \text { in } G(C) \text {. }
$$

Proof. Let $z \in \pi_{j}(Z), j \geqslant 4$. Then

$$
\begin{aligned}
\alpha_{m+1} \alpha_{m} z= & \alpha_{m+1}^{j+3} \alpha_{m}^{j} z=m\left\{(m+1) z \gamma^{2}+(-1)^{j+3}[\bar{c}, z \gamma]\right\} \\
& +(-1)^{j}\left\{(m+1)[\bar{c}, z] \gamma+(-1)^{j+3}[\bar{c}[\bar{c}, z]]\right\}=\otimes .
\end{aligned}
$$

Note that $2 \gamma^{2}=0$ eliminating the first term. Also $[\bar{c}, z \gamma]=\left[\bar{c}, z \gamma_{j}\right]=$ $[\bar{c}, z] \gamma_{j+3}$ since $\gamma$ a suspension (e.g. by 2.3 ) so $\otimes=(-1)^{j}[\bar{c}, z] \gamma-[\bar{c}[\bar{c}, z]]$. By above $\bar{c} \gamma_{4}=0$ so by $2.3,0=\left[z, \bar{c} \gamma_{4}\right]=(-1)^{7 j}\left[\bar{c} \gamma_{4}, z\right]=(-1)^{j+1}[\bar{c}, z] \gamma+$ $[\bar{c}[\bar{c}, z]]$ proving the claim.

Thus for any integer $m$ we have a stable secondary operation $\Phi_{m}$, $\Phi_{m}^{n}$ : $\pi_{n}(Z) \rightarrow \pi_{n+7}(Z), Z \in \operatorname{Top}^{c}, n \geqslant 9$, with $\operatorname{Dom} \Phi=\operatorname{Ker} \alpha_{m}$ and Ind $\Phi=$ $\operatorname{Im} \alpha_{m+1}$.

Example 2. Take $C=C P^{2}$. Let $c: S^{2}=C P^{1} \subset C P^{2}$ so $c \in \pi_{2}\left(C P^{2}\right)$ and $c \eta=0$ in $\pi_{3}\left(C P^{2}\right)$ where $\eta=\eta_{2}: S^{3} \rightarrow S^{2}$ is the Hopf map.

Define $\alpha, \beta \in G_{1}(C)$ by $\alpha, \beta: \pi_{j}(Z) \rightarrow \pi_{j+1}(Z), \alpha^{j}(z)=(-1)^{j}[\bar{c}, z], \beta^{j}(z)$ $=z \eta+(-1)^{j}[\bar{c}, z]$. Here $\eta=\eta_{j}=\Sigma^{j-1} \eta_{2}$ and $\bar{c}=\check{z}_{*} c_{1}(Z, \bar{z}) \in$ Top ${ }^{c}$. As above one can prove $\beta \alpha=0$ and $\alpha \beta=0$ in $G(C)$.

This gives stable operations $\Phi, \Psi: \pi_{n}(Z) \rightarrow \pi_{n+3}(Z), n \geqslant 5, Z \in$ Top $^{C}$, $\operatorname{Dom} \Phi=\operatorname{Ker} \beta$, Ind $\Phi=\operatorname{Im} \alpha$, Dom $\Psi=\operatorname{Ker} \alpha$, and $\operatorname{Ind} \Psi=\operatorname{Im} \beta$.

6. Some computations. Here we state two special cases of a general theorem on $[X, Y]^{A}$ and deduce some consequences. The general result gives a filtration of $[X, Y]^{A}$ in terms of homotopy groups and twisted operations. The statement is dual to that of $[9$, p. 211] however the proof is not dual. The method of transfer of category in [9] fails. The homotopy results needed for the transfer are due to the first author [7], [8]. The general result will be discussed in a separate paper.

Let $Q P^{n}$ be quaterionic projective space, suppose $f: Q P^{n-2} \rightarrow Z$ is given with a fixed extension $\bar{f}: Q P^{n} \rightarrow Z$

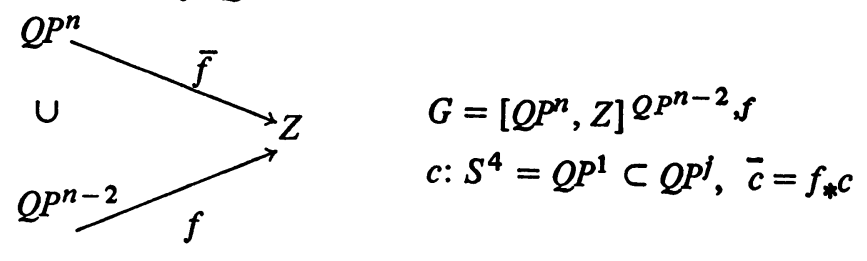




\subsection{THEOREM. There is an exact sequence of abelian groups}

$$
0 \rightarrow \operatorname{coker}\left(\alpha^{4 n-3}\right) \rightarrow G \rightarrow \operatorname{Ker}\left(\alpha^{4 n-4}\right) \rightarrow 0
$$

where

$$
\alpha^{j}: \pi_{j}(Z) \rightarrow \pi_{j+3}(Z), \alpha^{j}(z)=(n-1) z \gamma+(-1)^{j}[\bar{c}, z] .
$$

This is a 2-stage theorem. Theorems of this sort are proved by Nomura [10]. The version [11] does not contain the results-but the methods are dual. Our methods are different from Nomura's and have the advantage of readily extending to $k$-stage results.

Let us call a space $Z$ a $W_{k}$-space if $\left[z, z^{\prime}\right]=0$ for all $z \in \pi_{j}(Z), j<k$.

6.2 Corollary. Let $Z$ be a $W_{4}$-space. Then the order of $G$ is the same for any $f$ which extends to $Q P^{n}$.

In the above corollary and also for any $Z, f$ with $f_{*} c=0$ the operations are untwisted. This is not our main concern but we state one specific result for that case.

6.3 CoRollary. $Z=S^{4 n-3}, f=*$. Then $G=Z_{u(n)}, u(n)=\operatorname{gcd}(n-1,24)$, $n>2$.

Proof. This is immediate from 6.1.

Most of 6.3 is actually due to $R$. West [14, Theorem 14.1]. The reason is that $G=\left[Q P^{n}, S^{4 n-3}\right]=\pi^{4 n-3}\left(Q P^{n}\right)$ in this case. However 6.3 does settle ambiguities in West's calculation $(n \equiv 1,3(\bmod 6))$. It disagrees with his results in the cases $n \equiv 7,19(\bmod 24)$. His results for other dimensional spheres follow from 6.1 also.

6.4 Corollary. $Z=Q P^{n}, f=$ inclusion, $\bar{f}=$ identity. There is an exact sequence of abelian groups

$$
0 \rightarrow \operatorname{coker} \beta^{4 n-4} \rightarrow G \rightarrow \operatorname{Ker} \beta^{4 n-5} \rightarrow 0
$$

where $\beta^{j}: \pi_{j}\left(S^{3}\right) \rightarrow \pi_{j+3}\left(S^{3}\right), \beta^{j}(v)=(n-1) v \gamma+(-1)^{j}\left\langle\iota_{3}, \nu\right\rangle$, where $\langle$, denotes the Samelson product in $S^{3}$.

Proof. This follows from 6.1 and the results in [2] on Whitehead products in $Q P^{n}$.

Let $C P^{n}$ denote complex projective space and suppose $f: C P^{2} \rightarrow Z$ is given with a fixed extension $\bar{f}: C P^{5} \rightarrow Z$.

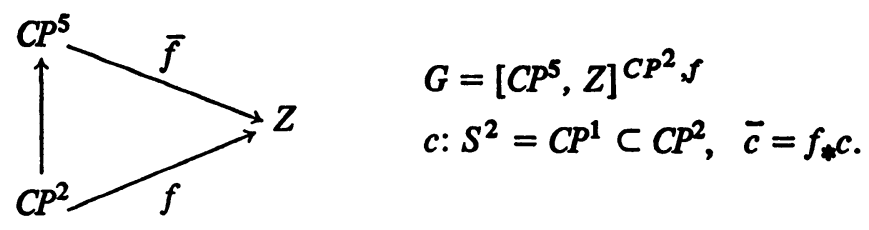


6.5 THEOREM.

$$
G=L_{0} \supset L_{1} \supset L_{2} \supset L_{3}=0, \quad L_{0} / L_{1}=\operatorname{Ker} \Phi \subset \pi_{6}(Z),
$$

$L_{1} / L_{2}=\operatorname{Ker} \alpha / \operatorname{Im} \beta \quad$ (subquotient of $\pi_{8}(Z)$ ), $\quad L_{2} / L_{3}=\pi_{10}(Z) / \operatorname{Im} \Phi$.

Here $\alpha, \beta, \Phi$ are stable $C P^{2}$-operations see $\S 5$, Example 2, for $Z \in$ Top $^{C}$, $C=C P^{2}$.

$$
\begin{array}{ll}
\alpha^{j}: \pi_{j}(Z) \rightarrow \pi_{j+1}(Z), & \alpha^{j}(Z)=(-1)^{j}[\bar{c}, z], \\
\beta^{j}: \pi_{j}(Z) \rightarrow \pi_{j+1}(Z), & \beta^{j}(z)=z \eta+(-1)^{j}[\bar{c}, z], \\
\Phi^{j}: \pi_{j}(Z) \rightarrow \pi_{j+3}(Z), & \operatorname{Dom} \Phi=\operatorname{Ker} \beta, \quad \operatorname{Ind} \Phi=\operatorname{Im} \alpha .
\end{array}
$$

Let $S^{j+3} \rightarrow^{u} K(\beta) \leftarrow S^{j}$ represent $\Phi$ (see Proof of 5.2, $u_{j}$ is $\tilde{\alpha}^{j}$ ). We will need two properties of $\Phi$ which come from the Proof of 6.5 .

(1) Consider the pushout diagram

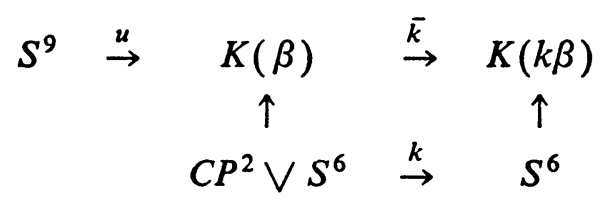

Then $\bar{k} u=i_{*} \nu$ in $\pi_{9}\left(S^{6} \cup_{\eta} e^{8}\right)$ where $\nu$ is the generator of the 2-component of $\pi_{9}\left(S^{6}\right)$. (2) Set $W=K(u)=C P^{2} \vee S^{6} \cup e^{8} \cup e^{10}$. Then in $H^{*}(W ; Z)$ we have $y w_{6}=w_{8}, y^{2} w_{6}=w_{10}$ where $y, y^{2}$ come from $H^{*}\left(C P^{2} ; Z\right)$ and $w_{8}, w_{10}$ are generators from the attached cells.

6.6 COROLlary. Suppose $f=g q$ where $q: C P^{2} \rightarrow S^{4}$ is the natural quotient map. Then $G=L_{0} \supset L_{1} \supset L_{2} \supset L_{3}=0$ where $L_{0} / L_{1}=\operatorname{Ker} \eta^{*} \cap \operatorname{Ker} \phi \subset$ $\pi_{6}(Z), \quad L_{1} / L_{2}=\pi_{8}(Z) / \operatorname{Im} \eta^{*}, \quad L_{2} / L_{3}=\pi_{10}(Z) / \phi\left(\operatorname{Ker} \eta^{*}\right)$. Here $\eta_{n} \in$ $\pi_{n+1}\left(S^{n}\right)$ is the nonzero element and $\phi^{j}: \pi_{j}(Z) \rightarrow \pi_{j+1}(Z)$ is the primary $S^{4}$-operation defined by $\phi(z)=\nu^{*} z+(-1)^{j}[g, z]$ where $\nu$ is the generator of the 2-component of $\pi_{j+3}\left(S^{j}\right)$.

Proof. Under the hypotheses we get $\alpha=0, \beta=\eta^{*}$ which gives the result for $L_{1} / L_{2}$. The other statements follow from the following claim: $\Phi^{j}=$ $\phi^{j} \mid \operatorname{Ker} \beta^{j}$. It will suffice by stability to prove the claim for $\Phi^{6}$. Consider

$$
\begin{array}{ccccc}
S^{9} & \stackrel{\mu^{6}}{\rightarrow} & K(\beta) & \rightarrow & P \\
& & \uparrow & & \uparrow \\
S^{7} & \rightarrow & C P^{2} \vee S^{6} & \stackrel{q \vee 1}{\rightarrow} & S^{4} \vee S^{6}
\end{array}
$$

Under the hypotheses of the corollary we may replace $\Phi$ by the $S^{4}$ operation defined by $S^{9} \rightarrow P \leftarrow S^{6}$. Note that $\Phi$ and $\phi \mid \operatorname{Ker} \beta$ have the same domain and zero indeterminacy. Let $p_{4}: S^{4} \subset P, p_{6}: S^{6} \subset P$. It suffices to prove that $\Phi\left(p_{6}\right)=\nu^{*}\left(p_{6}\right)-\left[p_{4}, p_{6}\right]$. Here $\Phi\left(p_{6}\right)=v \in \pi_{9}\left(S^{4} \vee\left(S^{6} \cup_{\eta} e^{9}\right)\right)=\pi_{9}\left(S^{4}\right)$ 
$\oplus \pi_{9}\left(S^{6} \cup_{\eta} e^{8}\right) \oplus Z\left[p_{4}, p_{6}\right]$, so $v=0 \oplus v_{2} \oplus \lambda\left[p_{4}, p_{6}\right]$. By the first property of $\Phi$ we see that $v_{2}=p_{6} \nu$. The second property shows that in $T=P \cup e^{10}$ we have $t_{4} t_{6}=t_{10}$ and this proves that $\lambda=-1$. This proves the claim and the corollary.

6.7 COROLlaRY. If $f_{*} c=0$ and $Z$ is a $W_{4}$-space then $G=L_{0} \supset L_{1} \supset L_{2} \supset$ $L_{3}=0$ and $L_{0} / L_{1}=\operatorname{Ker} \eta_{*} \cap \operatorname{Ker} \nu^{*}, L_{1} / L_{2}=\pi_{8} Z / \operatorname{Im} z^{*}, L_{2} / L_{3}=$ $\pi_{10}(Z) / \nu^{*}\left(\operatorname{ker} \eta_{*}\right)$.

Note that in the first example $f_{*} c=0$ sufficed to make all operations untwisted but here more is required.

Now take $Z=Q P^{2}$ and $f=g q, g: S^{4}=Q P^{1} \rightarrow Q P^{2}$. It is not hard to show that if $g=k s, k=k$ id: $Q P^{1} \rightarrow Q P^{1}, s: Q P^{1} \subset Q P^{2}$ and $24 \mid k(k-1)$ then $f$ will extend to $C P^{5}$.

6.8 COROLlary. $f=s k q$ as above. Then

$$
\left[C P^{5}, Q P^{2}\right]^{C P^{2} f}= \begin{cases}0 & k \equiv 1,2 \bmod 3 \\ Z_{3} & k \equiv 3 \bmod 3 .\end{cases}
$$

PRoof. Use 6.6. $i \leqslant 10$, so we have $\pi_{i-1}\left(S^{3}\right) \rightarrow^{\Sigma} \pi_{i}\left(S^{4}\right) \stackrel{s_{*}}{\rightarrow} \pi_{i}\left(Q P^{2}\right)$ where $s_{*} \Sigma: \pi_{i-1}\left(S^{3}\right) \cong \pi_{i}\left(Q P^{2}\right)$. Here $\alpha=0, \beta=\eta^{*}$. Both of these commute with $s_{*} \Sigma$ so can be computed using Toda's results [13]. The conclusion is $L_{0} / L_{1}=$ $0=L_{1} / L_{2} . \phi$ is not difficult to compute using Hilton [5], giving $L_{2} / L_{3}=0$, $k \equiv 1,2, L_{2} / L_{3}=Z_{3}, k \equiv 0$.

\section{REFERENCES}

1. W. D. Barcus and M. G. Barrat, On the homotopy classification of the extensions of a fixed map, Trans. Amer. Math. Soc. 88 (1958), 57-74.

2. M. G. Barrat, I. M. James and N. Stein, Whitehead products and projective spaces, J. Math. Mech. 9 (1960), 813-819.

3. A. L. Blakers and W. S. Massey, The homotopy groups of a triad. II, Ann. of Math. (2) 55 (1952), 192-201.

4. ___ Products in homotopy theory, Ann. of Math. (2) 58 (1953), 295-324.

5. P. J. Hilton, A certain triple Whitehead product, Proc. Cambridge Philos. Soc. 50 (1954), 189-197.

6. On the homotopy groups of the union of spheres, J. London Math. Soc. 30 (1955), $154-172$.

7. D. E. Kruse, Relative homotopy classification of maps, Thesis, Univ. of Kansas, 1974.

8. __ Some properties of relative principal cofibrations (submitted).

9. J. F. McClendon, Higher order twisted cohomology, Invent. Math. 7 (1969), 183-214.

10. Y. Nomura, A non-stable secondary operation and homotopy classification of maps, 1968 (preprint).

11. A non-stable secondary operation and homotopy classification of maps, Osaka J. Math. 6(1969), 117-134.

12. E. H. Spanier, The homotopy excision theorem, Michigan Math. J. 14 (1967), 245-255. 
13. H. Toda, Compasition methods in the homotopy groups of spheres, Princeton Univ. Press, Princeton, N. J., 1962.

14. R. W. West, Some cohomotopy of projective space, Indiana Univ. Math. J. 20 (1971), 807-827.

15. J. H. C. Whitehead, On adding relations to homotopy groups, Ann. of Math. (2) 42 (1941), $409-428$.

16. __ On certain theorem of G. W. Whitehead, Ann. of Math. (2) 58 (1953), 418-428.

Department of Mathematics, University of Kansas, LaWrence, Kansas 66044 